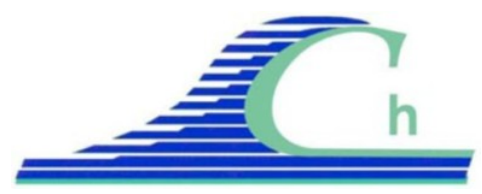

XII ${ }^{\text {èmes }}$ Journées Nationales Génie Côtier - Génie Civil

Cherbourg, 12-14 juin 2012

DOI:10.5150/jngcgc.2012.097-Y @ Editions Paralia CFL

disponible en ligne - http://www.paralia.fr - available online

\title{
Mesures de réduction des impacts du port Tanger Med II sur les ressources vivantes
}

\author{
Fatima YAHYA ${ }^{1}$ \\ 1. Société Tanger Med Engineering (TME), Zone franche Ksar Al majaz, Tanger, \\ Maroc. \\ f.yahya@tme.ma
}

\section{Résumé :}

L'implantation des infrastructures portuaires engendre des risques d'impact négatif sur l'environnement marin et naturel fragile. Ces projets doivent être accompagnés par des mesures d'accompagnement et de protection pour qu'ils puissent s'intégrer dans un processus de développement durable. C'est dans ce cadre que s'inscrit le complexe portuaire Tanger Med II qui devient une composante importante dans la stratégie de renforcement de la plateforme globale logistique et industrielle en cours de déploiement au Nord du Maroc, en tirant profit de la situation stratégique du détroit de Gibraltar.

Des études menées à grande échelle sur la Méditerranée ou bien sur des secteurs analogues, permettent d'indiquer que des cétacés, tortues marines et poissons de grandes valeurs écologiques et économiques sont présents dans les eaux de l'aire d'étude.

L'impact global du projet sur ces ressources marines vivantes est dû essentiellement aux activités de dragage et clapage. Cet impact est accentué par le trafic général dans le détroit et par le bruit émis à travers des ondes acoustiques.

Des mesures préventives et de compensation sont mises en place par le maître d'ouvrage et les entreprises chargées de l'exécution des travaux avant et pendant l'exploitation du port. Des programmes de suivi et de surveillance sont également mis en œuvre et intégrés dans la conception du projet afin de compenser les effets négatifs sur ces ressources vivantes.

Cette publication a pour but de partager une expérience pratique en matière de contribution à la protection des ressources naturelles afin d'éviter toute forme de gaspillage ou de dégradation susceptible de compromettre le développement durable.

Mots clés : Impact environnemental - Ressources vivantes marines - Reptiles Cétacés - Ichtyofaune - Pollution - Rejets liquides - Suivi environnemental - Mesures de préservation

\section{Introduction}

Le complexe portuaire Tanger Med II s'inscrit dans le cadre d'une stratégie de renforcement de la plateforme globale logistique et industrielle en cours de déploiement au Nord du Maroc, en tirant profit de la situation stratégique du détroit de Gibraltar. 
Le projet est situé dans la région Tanger Tétouan qui présente une position géostratégique d'importance, au niveau du détroit de Gibraltar. Les écosystèmes sont fragilisés par des exploitations abusives des ressources forestières, des carrières d'extraction de matériaux incontrôlées, une chasse démesurée de la faune sauvage et une pollution des nappes phréatiques de la dorsale calcaire.

L’implantation des infrastructures du port a été accompagnée par des mesures préventives, réductrices et compensatoires pour atténuer, voire supprimer l’impact négatif du projet sur l'environnement naturel fragile.

\section{Milieu biologique marin}

\subsection{Reptiles}

La zone Tanger Med est un lieu de passage privilégié des trois tortues marines suivantes :

- Tortue luth (Dermochelys coriacea). Cette espèce est présente en Méditerranée bien que rarement observée, elle ne semble pas nidifier sur ces côtes, mais elle les fréquente lors de leurs migrations entre l’Atlantique et la Méditerranée.

- Tortue verte (Chelonia mydas). Cette espèce est surtout présente en Méditerranée orientale bien qu'il y ait quelques sites de ponte en Turquie et à Chypre. Par ailleurs, la seule autre zone de contact connue à l'heure actuelle entre les tortues vertes de l'Atlantique- Méditerranée et les tortues vertes de l'Indopacifique est la côte Ouest de l'Afrique. Il est donc fort probable que la zone d'étude constitue une zone de passage pour cette tortue.

- Tortue caouanne (Caretta caretta). Le détroit de Gibraltar et la mer d'Alboran sont des lieux de passage privilégiés pour cette tortue qui migre, de l'Atlantique à la Méditerranée au début du printemps et, de la Méditerranée à l'Océan Atlantique pendant l'été et l'automne. L'aire du détroit sert de zone de recrutement de jeunes de cette espèce faisant augmenter les populations de façon notable durant les mois d'avril à juin. Les valeurs minimales se produisent durant les mois froids de décembre à avril.

\subsection{Cétacés}

La communauté de cétacés est considérée comme étant la mieux conservée de la zone méditerranéenne. L'aire côtière a une importance particulière pour cette communauté.

Les espèces de cétacés habituellement rencontrées dans le détroit de Gibraltar sont présentes tout au long de l'année avec une préférence pour la saison printanière. Les espèces les plus communément observées sont les dauphins bleus et blancs.

D’autres espèces de cétacés rencontrées occasionnellement dans le détroit de Gibraltar sont récapitulées dans le tableau 1. 


\section{XII ${ }^{\text {èmes }}$ Journées Nationales Génie Côtier - Génie Civil}

Cherbourg, 12-14 juin 2012

Tableau 1. Espèces de cétacés fréquentes dans le détroit de Gibraltar (d'après CASTELLS \& MAYO, 1993 ; DE STEPHANIS et al., 2005 ; FIRMM, site web).

\begin{tabular}{ll}
\hline Nom scientifique & Nom commun \\
\hline Stenella coeruleoalba & Dauphin bleu et blanc \\
\hline Delphinus delphis & Dauphin commun \\
\hline Tursiops truncatus & Grand dauphin \\
\hline Pseeudorca crassidens & Faux-orque \\
\hline Orcinus orca & Orque \\
\hline Globicephala melas & Globicephale noir \\
\hline Physeter macrocephalus & Cachalot \\
\hline Balaenoptera physalus & Rorqual commun \\
\hline Phocoena phocoena & Marsouin \\
\hline
\end{tabular}

\subsection{Ichtyofaune}

L'aire d'étude comporte des espèces de thonidés tels que le thon rouge et l'espadon qui sont considérées comme importantes sur le plan naturel, mais aussi sur le plan économique.

\subsubsection{Thon rouge (Thunnus thynnus)}

Lors de sa $17^{\text {ème }}$ réunion, le comité scientifique de la Commission Internationale pour la Conservation des Thonidés de l'Atlantique (ICCAT) a recommandé l'adoption d'une approche de précaution en ramenant le quota de pêche de thon rouge en Méditerranée à 12.900 tonnes en 2011 au lieu de 13.500 en 2010.

\subsubsection{Espadon (Xiphias gladius)}

Les prises d'espadon réalisées en Méditerranée constituent 96 \% de celles du Maroc. Durant cette dernière décennie, les prises totales d'espadon ont diminué à partir de 1998, pour se stabiliser autour de 3500 tonnes jusqu'en 2004. Ce niveau de captures semble être déterminé à la fois par l'effort de pêche déployé et l'abondance de la ressource traversant les côtes marocaines.

Depuis 1991, l’ICCAT a multiplié les recommandations et établi des quotas de prises par pays, ainsi que des minima de taille et d’âge. Lors de la 17è réunion de l'ICCAT, les scientifiques ont annoncé un stock en bonne santé et recommandé le maintien du quota global à 13700 tonnes en 2011.

\section{Effets du projet sur les milieux naturels et les équilibres biologiques}

\subsection{Travaux de clapage et dragage}

L’impact global sur le milieu biologique marin est principalement lié aux actions de la machinerie des travaux de clapage et dragage provoquées spécialement par l'utilisation des machines lourdes. 


\subsubsection{Turbidité et matière en suspension}

La turbidité (mise en suspension particulaires) temporaire peut provenir des travaux de dragage et du clapage suite au déplacement des engins et la remise en suspension des sédiments dus aux hélices.

Les effets liés à la turbidité puis à la sédimentation (décantation des suspensions après perturbation) en milieu marin et dans le contexte portuaire existant de port Tanger Med II sont :

- un frein temporaire à la pénétration lumineuse dans les eaux : impact sur la production primaire et sur les quelques espèces sensibles à la turbidité ;

- une sédimentation des particules entraînant un effet sur les ressources marines ; néanmoins, au regard des conditions portuaires actuelles (au vu de l'agitation due, entre autres, à la construction du port), cet impact est limité.

\subsubsection{Bruit}

Cet impact est accentué par la gêne due au trafic général dans le détroit.

\subsection{Rejets liquides}

Tant la construction que l'exploitation des infrastructures produisent une série de rejets qu'il est nécessaire de qualifier et caractériser dans le but d'évaluer les éventuels impacts et de proposer les mesures préventives et correctrices nécessaires.

\subsubsection{Pendant les travaux}

Le principal impact sur le milieu marin provient du lessivage des quais et des pontons par temps de pluie. Ce lessivage provoque le rejet de particules fines dans le port et ses abords et augmente les niveaux de turbidité. De même, les rejets d'eaux sales ou chargées issues des travaux pourraient générer la même turbidité ou une pollution par les hydrocarbures.

Lors du déversement d'hydrocarbures dans l'eau, la fraction lourde forme un film irisé qui, s'il occupe une partie importante de la surface du bassin, peut diminuer la pénétration de la lumière et l’oxygénation de l'eau.

\subsubsection{Pendant l'exploitation du port}

Les hydrocarbures et micropolluants provenant des postes hydrocarbures ou des bateaux, la pollution bactérienne, les matières en suspension générées par la fréquentation des quais et le rejet de macrodéchets (emballages en matière plastique, débris de bois, bidons d'huile, déchets alimentaires, cordages...) peuvent polluer le milieu marin. 


\section{XII ${ }^{\text {èmes }}$ Journées Nationales Génie Côtier - Génie Civil \\ Cherbourg, 12-14 juin 2012}

\subsection{Eaux usées}

\subsubsection{Pendant les travaux}

Durant les travaux de construction du port, des eaux usées sont générées et ce proportionnellement à la main d’œuvre nécessaire à la bonne conduite des travaux.

\subsubsection{Pendant l'exploitation du port}

Dans le port, deux types d'eaux usées seront produites :

- eaux usées sanitaires produites par les employés du port, par le nettoyage des entrepôts et par les passagers ;

- eaux noires des bateaux amarrés dans le port.

\section{Mesures de préservation des milieux naturels}

\subsection{Mesures générales}

En ce qui concerne l'organisation générale des travaux, les mesures suivantes ont été mises en place :

- création d'un plan de management environnemental et d'un plan d'urgence pour l'environnement, avant le début des travaux, définissant le protocole d'action en cas d'incident environnemental ;

- tenue d'un carnet de bord par les entreprises en charge des travaux décrivant les opérations réalisées et mise de ce carnet à la disposition des autorités compétentes, en cas d'incident, le responsable des opérations avertit immédiatement les autorités compétentes ;

- rédaction d'un manuel d'utilisation des substances pulvérulentes dans le port, accompagné des mesures préventives à prendre telles que l'humidification des voies de transport, l'installation de conduites lors du déchargement de matériaux lorsque leur chute se produit à une hauteur supérieure à $2 \mathrm{~m}$, l'utilisation d'écrans coupe-vent et de déflecteurs à trémies, ainsi que l'utilisation de bâches pour recouvrir les chargements dans les moyens de transport ;

- désignation d'un responsable environnement sur le chantier chargé du respect des procédures de gestion des déchets, de veiller au comportement des personnels, et doté d'une capacité d'initiative réelle en cas d'incident technique susceptible de générer des nuisances ;

- établissement d'un programme de sensibilisation et de formation à l'ensemble du personnel sur site concernant les questions environnementales, instaurant des pratiques de respect de l'environnement, ainsi que des mesures de sécurité face à d'éventuels accidents environnementaux ;

- renforcement de l'administration régionale et locale dans la zone du projet par la création de nouveaux centres et services à préoccupations environnementales ( à titre 
d'exemple, Tanger Med Utilities est une filiale de le groupe Pizzorno, pionnier de la gestion des déchets ménagers et industriels en France, qui a été créée pour la concession des services d'assainissement liquide et solide, de gestion de déchets, d'hygiène et de propreté du complexe Tanger-Med) ;

- création d'un bureau d'attention à la population où seront recueillies et enregistrées les plaintes de la population locale liées à l'environnement, suite auxquelles le maître d’ouvrage prévoit la mise en place de mesures préventives, réductrices et compensatoires ;

- dépôt de la demande du maître d’ouvrage auprès des autorités pour que le complexe portuaire Tanger Med II fasse partie de l'ensemble de ports liés au système international "Green Award", de sorte que l’on encourage, au moyen de bénéfices, l'entrée de bateaux associés au système de qualité environnementale "Green Award". Ce système suppose des réductions entre 3 et $6 \%$ pour les services du Complexe Portuaire pour tous les bateaux certifiés par le système "Green Award".

\subsection{Mesures pour la préservation de la qualité de l'eau}

\subsubsection{Limitation de la turbidité des eaux}

Afin de limiter la turbidité des eaux, des instructions sont communiquées au personnel en charge des travaux de dragage et clapage :

- éviter la mise en suspension de particules dans l'eau par une approche en douceur du fond par la benne ;

- ne pas effectuer de travaux de dragage et de clapage lors d'épisodes de vents forts ou de tempêtes ;

- s’assurer de l'étanchéité des compartiments des chalands afin d'éviter les pertes de sédiments et vérifier la fermeture adéquate du fond du navire après chaque déversement ;

- limiter le taux de liquéfaction des matériaux afin qu'ils demeurent le plus solide possible, lorsque le chaland est complètement immobile ;

- remplir le chaland à 90\% de sa capacité maximale afin de réduire les risques de surverse ou débordement lors de l'acheminement vers la zone de dépôt.

\subsubsection{Limitation des apports de produits polluants dans la colonne d'eau}

Les mesures suivantes sont appliquées afin d'éviter le rejet de produits polluants dans le milieu marin :

- installation des dépôts de carburant, d’huile ou d'autres produits pétroliers, si nécessaire, à plus de $30 \mathrm{~m}$ du littoral afin de limiter les risques de contamination de l'eau ; quand cela n’est pas réalisable, des mesures particulières de protection seront prises ;

- utilisation d'engins conformes aux normes en vigueur ; 


\section{XII ${ }^{\text {èmes }}$ Journées Nationales Génie Côtier - Génie Civil \\ Cherbourg, 12-14 juin 2012}

- inspection journalière des engins afin de s'assurer de leur bon état de fonctionnement, cette mesure permet d'éviter les accidents, les fuites de carburants, d'huile et de graisse ;

- surveillance constante lors du ravitaillement des navires afin de réaliser cette opération selon les méthodes prescrites et d'appliquer la procédure en cas de déversement accidentel ;

- surveillance visuelle quotidienne pour s'assurer qu'aucune pollution ne se disperse dans le milieu marin, quand il y a suspicion de pollution, celle-ci est contrôlée par les dispositifs adéquats déjà présents sur Tanger Med I et si nécessaire, un barrage flottant amovible pourrait être déployé afin de confiner la zone sinistrée ;

- renseignement dans un carnet de bord et information du responsable environnement des éventuelles fuites dues à des vannes déficientes ou à des erreurs humaines ;

- interdiction de déverser des rebuts, des hydrocarbures ou des produits dangereux, dans le plan d'eau ;

- récupération des eaux usées issues des engins de travaux (pompes amovibles ou pompes fixes ...);

- sensibilisation de toute personne étant amenée à travailler avec le système de ravitaillement : formation TMD (transporter des marchandises dangereuses) ou autre formation appropriée à la tâche ;

- communication et affichage de la procédure d'intervention en cas de déversement pour l'ensemble du personnel concerné ;

- mise en place des matériaux adéquats pour éponger et contenir les déversements accidentels provenant des équipements.

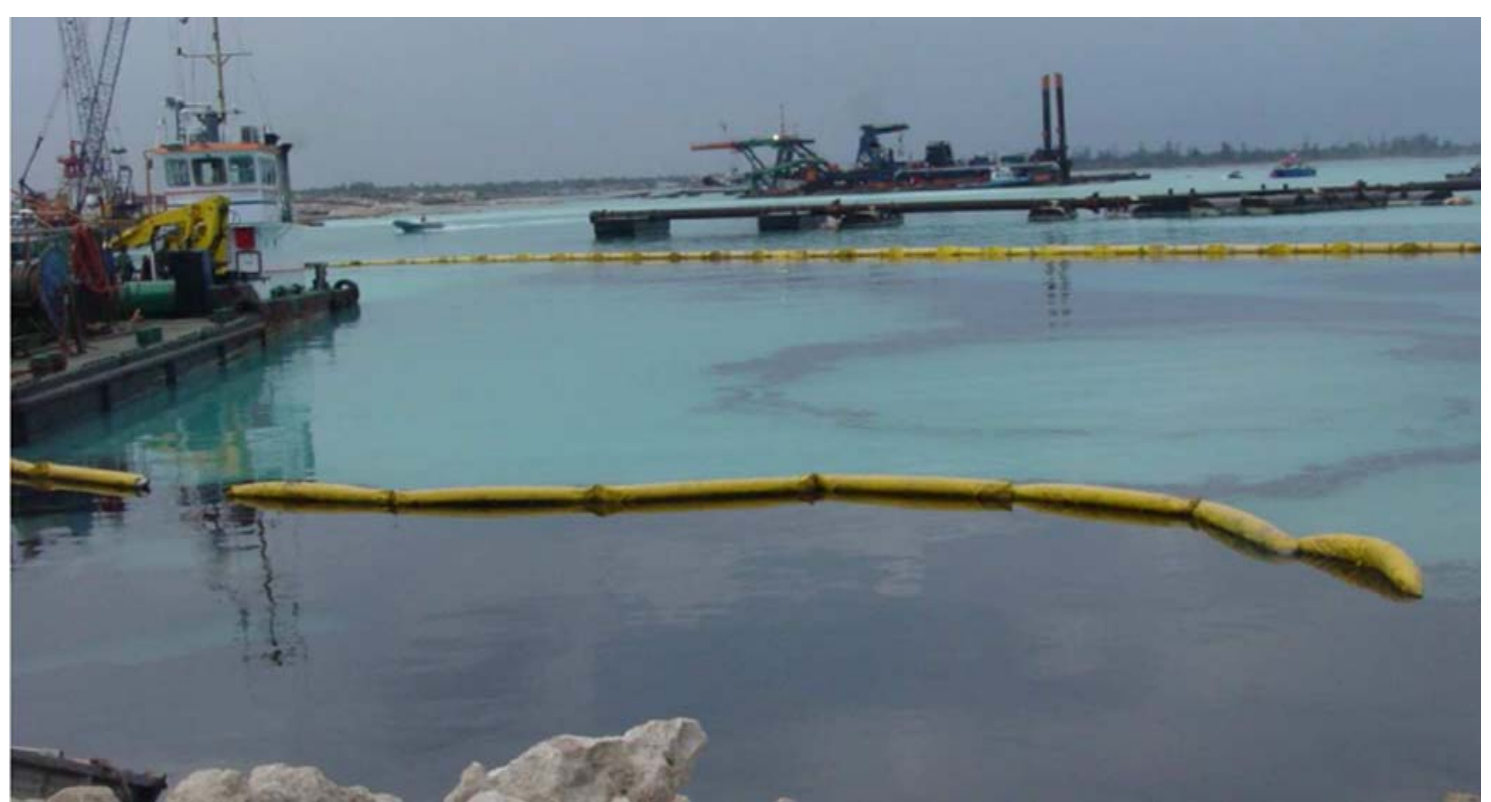

Figure 1. Barrages flottants canalisant les matières polluantes vers des poches amovibles jetables. 


\subsection{Mesures pour atténuer le bruit}

Des mesures de prévention, compensatoires et/ou correctrices permettant d'atténuer l'impact acoustique du projet ont été mises en place :

- établissement d'une procédure recueillant les normes concernant les bonnes pratiques environnementales pour réduire les niveaux sonores des bateaux qui mouillent dans le port ;

- réalisation de mesures des niveaux sonores dans toutes les agglomérations où l'on prévoit des niveaux sonores élevés, dûs au Projet Tanger-Med II, afin de vérifier l'efficacité des mesures adoptées pour limiter les niveaux du bruit, ces mesures sont réalisées conformément aux programmes de surveillance et de suivi environnemental établis par les entreprises chargées de l'exécution des travaux et communiqués au maître d'ouvrage pour approbation ;

- dans les cas où les niveaux sonores légiférés ou recommandés par les normes internationales de référence sont dépassés, des systèmes pour la réduction du bruit sont installés. Les mesures sont généralement des écrans antibruit, des billons, l'insonorisation ou l'isolement de sources de bruit, la modification de la distribution du trafic, la limitation d'activités aux différents horaires nocturne et diurne, la prohibition d'activités bruyantes spécifiques.

\subsection{Mesures pour atténuer les pollutions lumineuses}

Les interventions d'éclairage architectural du projet respectent les indications insérées dans les projets de construction de manière à limiter les pollutions lumineuses.

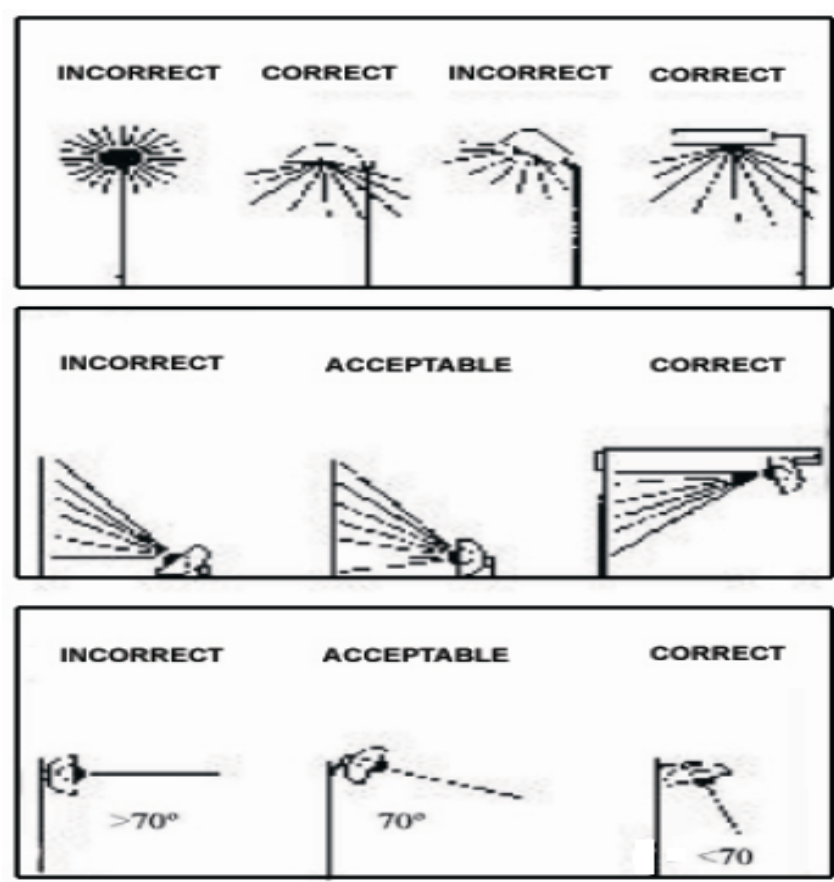

Figure 2. Recommandation pour limiter les pollutions lumineuses. 


\section{XII ${ }^{\text {èmes }}$ Journées Nationales Génie Côtier - Génie Civil \\ Cherbourg, 12-14 juin 2012}

\subsection{Mesures en cas de pollution accidentelle}

Dans le cadre des projets de construction de Tanger Med, l’Agence Spéciale Tanger Méditerranée (TMSA) a mis en place un plan d'urgence de prévention et de lutte contre la pollution accidentelle par des hydrocarbures dans le Complexe Portuaire Tanger Med (PUPTM).

L'objectif de ce plan est d'empêcher, notamment par des actions de prévention, et de limiter autant que possible les conséquences d'une pollution maritime accidentelle au port de Tanger Med qui surviendrait au cours des activités normales d'exploitation portuaire. Il est donc destiné à :

- définir l'organisation de la lutte anti-pollution pour permettre une réponse rapide adaptée à l'importance de la crise et aux conséquences prévisibles de l'incident ainsi que l'interface avec d'autres plans en particulier le plan d'urgence national de lutte contre la pollution, les plans des terminaux concédés, le plan du port de Tanger ville et ceux des ports européens de l'autre rive du détroit de Gibraltar ayant des conventions de partenariat ou de jumelage avec le port de Tanger Med ;

- préciser le rôle et les missions des responsables du port de Tanger Med II en charge de la lutte contre la pollution par hydrocarbures ou autres produits chimiques présentant un risque pour l'environnement marin ou terrestre du port ;

- décrire les premières mesures à prendre dès constatation de la pollution ;

- définir des stratégies de lutte en fonction des scénarios d'accidents ;

- identifier les moyens humains et matériels disponibles et donner des informations sur les processus de formation et de simulation du personnel chargé de la lutte antipollution ;

- préciser le mécanisme de la gestion de l’après-crise.

\section{Mesures de surveillance et de suivi}

\subsection{Surveillance des impacts marins en période de travaux}

Un suivi de la fréquentation de la zone par les cétacés, les tortues et les thons rouges est réalisé avant, pendant et après les travaux.

Pour éviter les collisions et les impacts des moyens de surveillance simples sont mis en place :

- établissement du contact avec la capitainerie et les organisations non gouvernementales locales pour savoir si des observations récentes ont été effectuées ;

- observation du large avec des jumelles pour détecter les cétacés ou reptiles marins à des distances importantes. Dès lors qu'un cétacé est observé à $1 \mathrm{~km}$ de la zone de dépôt, les personnes en charge de la surveillance sont amenées à notifier l'autorité maritime et les garde-côtes et diminuer l'intensité des travaux. 
5.2 Mise en place d'une procédure de suivi de bilan des engagements

Les objectifs sont d'une part d'organiser le contrôle du respect des engagements du maître d'ouvrage en matière d'environnement, d'autre part d'évaluer les effets réels de l'opération au bout d'un certain temps :

- évaluer les effets réels, prévus ou non, positifs et négatifs de l’opération, constater l'efficacité des moyens mis en œuvre, et expliquer les différences constatées ;

- améliorer la connaissance des effets du projet portuaire sur l'environnement afin de capitaliser le savoir-faire en matière de maîtrise des impacts sur l'environnement.

Un comité de suivi des engagements environnementaux composé des administrations, professionnels maritimes, établissements d'océanologie, communes et associations, se réunit périodiquement pour discuter du bilan environnemental comprenant :

- évaluation de l'incidence des travaux sur le milieu naturel et la proposition des mesures d'atténuation aux impacts réels sur les espèces vivantes par le constat de l'emprise réelle du chantier dans sa globalité ;

- suivi de la mise en œuvre des mesures compensatoires sur les milieux naturels suite aux réunions tenues auparavant ;

- suivi de l'application du cahier de charge environnemental et du plan de management de l'environnement.

\section{Conclusion}

Le chantier Tanger Med II est en cours avec un avancement global à fin Janvier 2012 de $40 \%$ environ. Conjointement à l'avancement des travaux, le maître d'ouvrage et les entreprises chargées de l'exécution des travaux prennent les dispositions nécessaires pour assurer le suivi et la surveillance de l'environnement pour protéger les ressources naturelles contre toute forme de gaspillage ou de dégradation potentielle.

La mise en valeur du patrimoine naturel est considérée comme une des grandes actions environnementales volontaires des parties prenantes. Pour affirmer la volonté de consacrer le site comme une implantation respectueuse d'un développement durable, un concept de renaturation du site est mis en œuvre sur les premiers secteurs d'exploitation du complexe portuaire. Le budget engagé par TMSA dans ce projet est évalué à près de 30 millions de Dirhams.

\section{Références}

CASTELLS A., MAYO M. (1993). Guia de los mamiferos en libertad de espana y Portugal. Madrid : Ediciones Piramide.

DE STEPHANIS R., VERBORGH P., PEREZ GIMENO N., SANCHEZ CABANES A., PEREZ JORGE S., ESTEBAN PAVO R., SELLER N., URQUIOLA E., GUINET C. (2005). Impacts produits par le trafic maritime sur les populations de cétacés dans le détroit de Gibraltar. Situation actuelle et prévisions de futur. Document interne CIRCE. FIRMM (site web). http://www.Firmm.org 